\title{
Evidence for Supercurrent Quantization in Interfacial Josephson Junctions
}

\author{
M.J. Black, B.W. Alphenaar甘, and H. Ahmed \\ University of Cambridge Microelectronics Research Centre and Hitachi Cambridge Laboratory, \\ Cavendish Laboratory, Madingley Road, Cambridge, CB3 OHE, UK
}

(November 4, 2018)

\begin{abstract}
We observe a series of discontinuities in the above gap current/voltage characteristics of highly transmissive $\mathrm{Nb} / \mathrm{Si}$ superconductor/semiconductor interfaces. The bias at which each switch occurs decreases in quantized steps as a function of temperature and magnetic field. The observed step-size is the same for both dependencies. We propose that the steps correspond to supercurrent quantization through granular Josephson junctions formed near the interface.
\end{abstract}


The conductance through a quantum point contact in a two-dimensional electron gas (2DEG) is quantized to $2 \mathrm{e}^{2} / \mathrm{h}$ times the number of transmitted one-dimensional channels [1]. Analogous behavior is predicted for a small area Josephson junction formed by a narrow constriction between two superconductors [2]. In this case, quantization is in the critical current, and is given by e $\Delta_{0} / \hbar$ times the number of transmitted one-dimensional channels, where $\Delta_{0}$ is the pairing potential of the superconductor. Recently, Takayanagi et al. [3] fabricated the first working superconducting quantum point contacts, using $\mathrm{Nb}$ contacts to a submicron width InAs $2 \mathrm{DEG}$, modulated by a split-gate. They observed steps in both the critical current and the normal conductance as a function of gate bias. However, substantial fluctuations were observed in the step height, and the critical current was not quantized at the theoretically predicted values. Muller et al. [- measured the conductance and critical current through a break junction of $\mathrm{Nb}$. They observed steps in the critical current consistent with the theoretically predicted value, suggesting that individual conductance channels are gradually shut off as the filament is broken. More recent experiments [5] imply, however, that such critical current steps might be caused by abrupt atomic rearrangements, which change the normal resistance [6].

This paper presents intriguing new evidence for the quantization of the critical current in small area Josephson junctions. We perform detailed measurements of the interface resistance of $\mathrm{Nb} / \mathrm{Si}$ junctions. Below a transition temperature of $1.5 \mathrm{~K}$, we observe a series of switches in the above gap DC current/voltage characteristics. As the temperature increases towards the transition, or as a magnetic field is applied, the switching bias decreases in a series of quantized steps. The observed step-size is equal in both temperature and magnetic field dependencies. We propose that the switches occur as we exceed the critical supercurrent of Josephson junctions formed between $\mathrm{Nb}_{3} \mathrm{Si}$ particles and the $\mathrm{Nb}$ contact. By varying the temperature or magnetic field we are able to reduce the junction area, and observe a corresponding quantized change in the critical current.

Fig. 1(a) shows a schematic drawing of our $\mathrm{Nb} / \mathrm{Si}$ junctions. A Nb contact is made to a $53 \mathrm{~nm}$ thick single crystal $\mathrm{n}^{++} \mathrm{Si}$ layer which has been wafer bonded to $100 \mathrm{~nm}$ of $\mathrm{SiO}_{2}$ on a $\mathrm{p}^{+} \mathrm{Si}$ substrate. $2.0 \mathrm{~K}$ Hall measurements of the Si layer indicate that the carrier density $n=5.3 \times 10^{25} \mathrm{~m}^{-3}$ and the elastic scattering length $l_{e}=10.8 \mathrm{~nm}$. We create a highly transmissive interface by first sputter-etching the Si surface at $200 \mathrm{~W}$ for 10 minutes in an Ar pressure of $3 \times 10^{-2}$ mbar. This removes a $25 \mu \mathrm{m} \times 25 \mu \mathrm{m}$ square of the (100) Si top-layer, and exposes clean (111) Si side-surfaces. Without breaking the vacuum we then deposit $180 \mathrm{~nm}$ of $\mathrm{Nb}$, resulting in a total $\mathrm{Nb} / \mathrm{Si}$ contact area of approximately $A_{c}=6$ $(\mu \mathrm{m})^{2}$. We measure the critical temperature of our $\mathrm{Nb}$ to be $T_{c}=9.0 \mathrm{~K}$, indicating a high quality film. Four-terminal measurements of the $\mathrm{Nb} / \mathrm{Si}$ junction resistance are made via $\mathrm{Ag}$ contacts to the $\mathrm{Si}$, and $\mathrm{Au}$ contacts to the $\mathrm{Nb}$. The effects we describe below have been observed in four different devices, each fabricated using the same method.

In the inset to Fig. 2, the differential resistance $(\mathrm{dV} / \mathrm{dI})$ of a typical junction is plotted as a function of applied bias. Measurements at three temperatures (1.6, 1.2 and $0.35 \mathrm{~K}$ ) are shown, normalized with respect to the resistance at high bias. In each trace, there is a broad dip in $\mathrm{dV} / \mathrm{dI}$ corresponding to the $\mathrm{Nb}$ gap energy $\left(\Delta_{0}= \pm 1.6 \mathrm{meV}\right)$. Further measurements (not shown) demonstrate that the dip survives up to the $\mathrm{Nb}$ transition temperature. This $\mathrm{dV} / \mathrm{dI}$ feature has been reported by a number of groups [7,8] and can be attributed to Andreev reflection at the $\mathrm{Nb} / \mathrm{Si}$ interface [9, [10]. In the $0.35 \mathrm{~K}$ trace we also observe a strong 
sub-gap resistance dip. (This first appears at temperatures below $1.1 \mathrm{~K}$.) A similar feature was reported by Heslinga et al. [7] and attributed to the appearance of a "proximity induced gap,' in the Si layer. More simply, the RSJ (resistively shunted junction) model predicts that a sub-gap dip can be observed if a Josephson junction forms in parallel with the interface resistance [11].

In our case, a possible explanation for the sub-gap resistance dip is suggested by the temperature dependence of $\mathrm{dV} / \mathrm{dI}$ at zero bias (not normalized), plotted in the main part of Fig. 2. The differential resistance decreases sharply at a temperature $T_{1}=1.5 \mathrm{~K}$, from 890 to $480 \Omega$. This drop, which strongly resembles a superconducting transition, occurs at the reported transition temperature of $\mathrm{Nb}_{3} \mathrm{Si}$ [12]. If we assume that the ratio between the transition temperature $T_{1}$ and the gap energy $\Delta_{N b_{3} S i}$ equals that for $\mathrm{Nb}$, then $\Delta_{N b_{3} S i}=0.25$ meV. This is similar to the observed resistance dip width of $\pm 0.28 \mathrm{mV}$ at $T=0.35 \mathrm{~K}$. These results imply that the sub-gap feature is due to Andreev reflection from $\mathrm{Nb}_{3} \mathrm{Si}$ particles that form near the interface during fabrication. $\mathrm{Nb}_{3} \mathrm{Si}$ has a high eutectic temperature $\left(645^{\circ}\right.$ $\mathrm{C})$, but it has been shown that $\mathrm{Nb}$ diffuses nonuniformly into $\mathrm{Si}$ at our maximum process temperature of $300^{\circ} \mathrm{C}$ (immediately following the sputter-etch), and forms small intermixed regions [13]. We also measured junctions made without the sputter-etch, and these showed no sub-gap dip or resistance transition.

Figure 3(a) gives results of a four-terminal DC measurement of the $\mathrm{Nb} / \mathrm{Si}$ interface (see inset) at 1.6 and $0.35 \mathrm{~K}$, corresponding to temperatures above and below the transition temperature, $T_{1}$. At $T=1.6 \mathrm{~K}$, the $\mathrm{I}-\mathrm{V}$ curve has a weak Schottky barrier dependence. At $T=0.35 \mathrm{~K}$, the current rises above the $1.6 \mathrm{~K}$ value, as expected from the results of Fig. 2. As the applied bias increases, the measured current and voltage switch discontinuously due to a sudden increase in the interface resistance. (A switch is observed in both the current and the voltage across the interface $V_{m}$ because only the total applied bias $V_{a}$ is fixed at each measurement point.) A total of six switches are observed with increasing bias, until the above and below transition I-V characteristics are almost identical. In Fig. 3(a), we also plot the $0.35 \mathrm{~K}$ characteristic measured with a transverse magnetic field of $B=$ $1.0 \mathrm{~T}$ (dashed line). The $\mathrm{I}-\mathrm{V}$ is almost identical to the above transition trace measured at $0 \mathrm{~T}$-only a small excess current is observed at low bias. We note that these results are completely reproducible, and that non-reproducible noise due to heating is not observed until the current $>200 \mu \mathrm{A}$. The maximum power through the device is $\sim 1 \mu \mathrm{W}$, while our pumped ${ }^{3} \mathrm{He}$ cryosat is able to maintain a $100 \mu \mathrm{W}$ heat load at $0.35 \mathrm{~K}$ for 3.5 hours.

In Fig. 3(b), we plot a series of $26 \mathrm{I}-\mathrm{V}$ characteristics for temperatures between 1.6 and $0.35 \mathrm{~K}$. The lowest lying trace corresponds to $T=1.6 \mathrm{~K}$. The traces above this correspond to the temperatures $1.55 \mathrm{~K}, 1.50 \mathrm{~K}, \ldots, 0.35 \mathrm{~K}$ and are offset by $0.25 \mu \mathrm{A}, 0.50 \mu \mathrm{A}, \ldots, 6.25 \mu \mathrm{A}$, respectively. Plotted in this way, the resistance switches reveal a remarkable temperature dependence. As the temperature increases from $0.35 \mathrm{~K}$, the bias at which each switch occurs decreases discontinuously, in a series of steps. For any particular switch, the step width is approximately constant. If we consider the three most clearly defined switches (marked $S_{1}$, $S_{2}$, and $S_{3}$ ), the average width of the first four steps is $d V_{1}=1.60 \mathrm{mV}, d V_{2}=1.82 \mathrm{mV}$ and $d V_{3}=2.04 \mathrm{mV}$, with a maximum variation (per switch) of $3 \%$. The steps can be seen more clearly if the switching bias $V_{n}$ is plotted as a function of temperature. This is shown in the inset of Fig. 3(b) where $V_{n}$ is plotted for the three switches $S_{1}, S_{2}$ and $S_{3}$ as a function of the reduced temperature $T / T_{1}$. The biases are normalized by the average step widths, $d V_{n}$. In 
each case, three or more equally spaced steps are observed. As the temperature approaches $T_{1}$, the switches merge together.

Further measurements provide more convincing evidence for the quantization of the switching bias. Figure 4(a) shows the normalized switching bias as a function of temperature taken from observations of the lowest bias switch in a second device. These points are compiled from a set of $94 \mathrm{I}-\mathrm{V}$ measurements (similar to those shown in Fig. 3(b)) taken at equally spaced temperatures between 1.30 and $0.37 \mathrm{~K}$. A total of 12 well-defined steps are observed, each containing at least three bias points. The average step width, $d V$, is 0.42 $\mathrm{mV}$. We point out that 4 other switches were observed in this device, and each showed a similar temperature dependence, although $d V$ is different for each switch.

We also studied the influence of magnetic field on the switching bias. Figure 4(b) shows the normalized switching bias for the switch shown in Fig. 4(a) as a function of magnetic field perpendicular to the current path. These points were compiled from a set of $130 \mathrm{I}-\mathrm{V}$ measurements taken at magnetic fields equally spaced between 0 and 0.33 Tesla $(T=0.37$ $\mathrm{K})$. The data clearly show that the switching bias is also quantized as a function of magnetic field. We observe 12 well-defined steps each containing 6 or more $B$-field points. The bias is again normalized using $d V=0.42 \mathrm{mV}$. For $B>0.25 \mathrm{~T}$, the steps dissapear, and the switching bias decreases contiuously with magnetic field. Most remarkably, a comparison between Figs. 4(a) and (b) shows that the quantized step-size is equal to the same value in the $B$-dependence as in the $T$-dependence. The background $T$ and $B$-dependences, however, are clearly different. Similar results were observed in all 5 switches of this device.

To understand these results, we now consider the $\mathrm{Nb} / \mathrm{Si}$ interface in more detail (see Fig. 1(b)). Current flows across the interface by electron tunneling through the Si depletion region. Because of the high doping density, the average inter-dopant spacing $\left(n^{-1 / 3} \approx\right.$ $2.7 \mathrm{~nm})$ is comparable to the depletion width $(3.7 \mathrm{~nm})$ [14]. In this case, it has been shown that the depletion width varies substantially across the contact and significant transmission occurs only at the most transparent sections of the interface [15]. We calculate the approximate area of the transparent regions as follows. The average transmission coefficient per quantum channel is given by $t_{a v}=R_{S} / R_{j}$ where $R_{S}$ is the Sharvin resistance and $R_{j}$ is the measured junction resistance. From Fig. $2, R_{j}$ at $1.6 \mathrm{~K}=890 \Omega$, and $R_{S}$ for our contact is $20 \mathrm{~m} \Omega$ so that $t_{a v}=2.3 \times 10^{-5}$. On the other hand, a fit of the normalized $\mathrm{dV} / \mathrm{dI}$ dip at $1.6 \mathrm{~K}$ to the BTK formalism gives a transmission coefficient for the transparent regions of $t_{A}=0.84$ [10]. The maximum area of the transparent regions is then approximately $A_{T}=A_{c} \times\left(t_{a v} / t_{A}\right)=164 \mathrm{~nm}^{2}$, where $A_{c}$ is the total contact area. Below the transition, at $T=1.2 \mathrm{~K}$, the resistance drops to $R_{j}=480 \Omega$, but the size of the normalized $\operatorname{dip}$ in $\mathrm{dV} / \mathrm{dI}$ remains roughly the same (see inset, Fig. 2). This means that $t_{A}$ stays constant, while the transmissive area increases to approximately $164 \mathrm{~nm}^{2} \times(890 \Omega / 480 \Omega)=304 \mathrm{~nm}^{2}$.

Additional conduction pathways below $T_{1}$ are created if $\mathrm{Nb}_{3} \mathrm{Si}$ particles form near the interface during sputtering. The pathlength for tunneling via the particles is reduced at the $\mathrm{Nb}_{3} \mathrm{Si}$ transition temperature, and the effective transmissive area increases. The crosssectional area for the $\mathrm{Nb}_{3} \mathrm{Si}$ particles is approximately equal to the increase in the transmissive area or $\approx 140 \mathrm{~nm}^{2}$. As shown in Fig. 1(b), if the normal coherence length $\xi_{n}$ reaches the $\mathrm{Nb}_{3} \mathrm{Si}$ particles a supercurrent can flow through weak-link Josephson junctions between the $\mathrm{Nb}_{3} \mathrm{Si}$ particles and the $\mathrm{Nb}\left(J_{1}\right.$ and $J_{2}$ in Fig. $\left.1(\mathrm{~b})\right)$. 
Consider now the switches observed in the I-V charactersitics of Fig. 3. The switches progressively transform the $T<T_{1}$ resistance $(0.35 \mathrm{~K})$ into the $T>T_{1}$ resistance $(1.6 \mathrm{~K})$ with increasing bias. Each switch is thus a partial breakdown of the conduction pathways added at $T_{1}$. This could be possibly explained by assuming that Joule heating raises the temperature of the conduction pathways above $T_{1}$. In this case [16], the switching bias should follow a $\left(T_{1}-T\right)^{1 / 2}$ dependence. Our observed switching bias, however, increases more slowly at low $T$ than this. In addition, it is unclear how a heating model can explain the quantization we observe in Fig. 4.

Resistance steps have also been reported by Hebard and Vandenberg in the I-V characteristics of high-resistance granular lead films [17]. These were later explained as the switching of rows of underdamped Josephson junctions formed between grains, lying perpendicular to the current path [18]. A switch occurs as a row moves from a zero-voltage state, to one in which a vortex undergoes a phase-slip process. The switches we observe might possibly be due to a similar process ocurring among granular Josephson junction arrays within the depletion region. Typically, though, the separation between switches in a voltage biased Josephson-junction array are independent of small magnetic field, and as the temperature is increased, the switches simply disappear [18,19] — quantization is not observed.

We might assume, then, that a group of Josephson junction pathways are associated with each particular switch. As the coherence length in the depletion region decreases with increasing $T$ or $B$, individual conduction channels are cut off, resulting in discrete steps. But since the observed step spacing is quantized for a particular switch, this would require an identical contribution from each random pathway.

We instead propose that the switches are due to the critical current transition of individual nanometer scale Josephson junctions formed between $\mathrm{Nb}_{3} \mathrm{Si}$ particles and the $\mathrm{Nb}$ contact. Consider a simple circuit model for the Nb/Si contact (Fig. 1(c)). Transport through the nth silicide particle is given by a Josephson junction $J_{n}$ in series with a $\mathrm{Nb}_{3} \mathrm{Si} / \mathrm{Si}$ interface resistance $R_{n}$. In the superconducting state, the Josephson junction resistance is zero, and the current through $J_{n}$ is simply $I_{n}=V / R_{n}$. The switching bias is thus proportional to the current carried by the Josephson junction at the critical current transition.

If the area of the Josephson junction is small enough, the switching bias should be quantized to $V=N e \Delta_{0} / R_{n} \hbar$, where $\mathrm{N}$ is the number of one-dimensional transmitted channels, and $e \Delta_{0} / R_{n} \hbar$ is equal to the quantized step size $d V$. In our case, $N=k_{F}^{2} A / 4 \pi$, where $A$ is the cross-sectional area of the junction. To produce the observed quantization, we assume that $A$ decreases as a function of $B$ and $T$. Since the normal coherence length $\xi_{n}$ decreases with increasing $B$ and $T$, this is reasonable. The number of modes $N$ in each junction (Fig. 3 and 4 ) is simply equal to the normalized switching bias $V / d V$. From the maximum number of modes, we can calculate the approximate cross-sectional area $A_{n}$ of the particles corresponding to the three switches $S_{1}, S_{2}$, and $S_{3}$ in Fig. 3. This gives $A_{1}=56 \mathrm{~nm}^{2}, A_{2}=84$ $\mathrm{nm}^{2}$ and $A_{3}=131 \mathrm{~nm}^{2}$, roughly corresponding to the cross-sectional area determined from our simple estimate above $\left(140 \mathrm{~nm}^{2}\right)$, and giving an average particle radius on the order of $\lambda_{F}$.

We expect that, in the limit of large $N$, the $T$ and $B$ dependences in Fig. 4 should be described by $I_{c}(T, B)$ for a large area point contact. The envelope of our quantized $T$ dependence appears similar to $I_{c}(T)$ for a large area device [20], while the envelope of the quantized $B$-dependence resembles the low field behavior of a screened Josephson junction 
[11]. The field required to insert a single flux quantum in such small junctions $(\sim 100$ $\mathrm{nm}^{2}$ ) is larger than the critical field of $\mathrm{Nb}$, so that no oscillatory dependence is expected. A detailed understanding would require a rigorous analysis of $I_{c}(T, B)$ in the crossover between macroscopic and nano-scale junctions.

In conclusion, we observe evidence that the maximum supercurrent through Josephson junction pathways formed near a $\mathrm{Nb} / \mathrm{Si}$ interface is quantized, in agreement with theoretical predictions. This could provide a new and straightforward way to study supercurrent transport in nanometer-scale Josephson junctions.

The authors thank F. Hekking, T. Klapwijk, M. de Jong, and D. Williams for valuable discussions and S. Newcomb for providing TEM analysis. 


\section{REFERENCES}

* To whom correspondence should be addressed.

[1] B.J. van Wees et al. Phys. Rev. Lett. 60, 848 (1988); D.A. Wharam et al., J. Phys. C 21, L209 (1988).

[2] C.W.J. Beenakker and H. van Houten, Phys. Rev. Lett. 66, 3056 (1991); A. Furusaki, H. Takayanagi, and M. Tsukada, ibid 67, 132 (1991).

[3] H. Takayanagi, T. Akazaki, and J. Nitta, Phys. Rev. Lett. 75, 3533 (1995).

[4] C.J. Muller, J.M. van Ruitenbeek, and L.J. de Jongh, Phys. Rev. Lett. 69, 140 (1992).

[5] C.J. Muller et al. Phys. Rev. B 53, 1022 (1996).

[6] T.N. Todorov and A.P. Sutton, Phys. Rev. Lett. 70, 2138 (1993).

[7] D.R. Heslinga et al. Phys. Rev. B 49, 10484 (1994).

[8] M. Hatano et al. Appl. Phys. Lett. 61, 2604 (1992); P.H.C. Magnée, PhD Thesis, University of Groningen, (1996).

[9] A.F. Andreev, Zh. Eksp. Teor. Fiz. 46, 1823 (1964) [Sov. Phys. JETP 19, 1228 (1964)].

[10] G.E. Blonder, M. Tinkham, and T.M. Klapwijk, Phys. Rev. B 25, 4515 (1982).

[11] M. Tinkham, Introduction to Superconductivity, (McGraw-Hill, New York, 1996).

[12] B.W. Roberts, Properties of Selected Superconducting Materials, (U.S. Government Printing Office, Washington, D.C., 1978).

[13] S.R. Mahamuni, D.T. Abell, and E.D. Williams, Solid State Comm. 68, 145 (1988).

[14] The depletion width is calculated using the CDMT model taking the Schottky barrier height to be $0.5 \mathrm{eV}$. See E.L. Wolf, Principles of Electron Tunneling Spectroscopy, (Oxford University Press, Oxford, 1989).

[15] E.L. Wolf and D.L. Lossee, Phys. Rev. B 2, 3660 (1970).

[16] W.J. Skocpol, M.R. Beasley, and M. Tinkham, J. Appl. Phys. 45, 4054 (1974).

[17] A.F. Hebard and J.M. Vandenberg, Phys. Rev. Lett. 44, 50 (1980).

[18] H.S.J. van der Zant et al., Phys. Rev. B 38, 5154 (1988).

[19] W. Yu and D. Stroud, Phys. Rev. B 46, 14005 (1992).

[20] I.O. Kulik and A.N. Omel'yanchuk, Fiz. Nisk. Temp. 3, 945 (1977); 4, 296 (1978) [Sov. J. Low Temp. Phys. 3, 459 (1977); 4, 142 (1978)]. 


\section{FIGURES}

FIG. 1. (a) Schematic drawing of our Nb/Si junctions. (b) A representative region of the $\mathrm{Nb} / \mathrm{Si}$ interface. $\mathrm{Nb}_{3} \mathrm{Si}$ particles, which form during $\mathrm{Nb}$ deposition, provide additional tunneling pathways. At low enough temperatures the normal coherence length $\xi_{n}$ extends to create Josephson junctions $J_{1}$ and $J_{2}$. (c) Circuit model of the $\mathrm{Nb} / \mathrm{Si}$ interface. The $\mathrm{Nb}_{3} \mathrm{Si}$ Josephson junctions $J_{n}$ have normal state resistances $R_{n}^{\prime}$ and lie in series with the $\mathrm{Nb}_{3} \mathrm{Si} / \mathrm{Si}$ interface resistances $R_{n}$. The in-parallel Schottky barrier resistance is $R$.

FIG. 2. Zero-bias differential resistance of a highly transmissive Nb/Si junction verses temperature. $T_{1}$ is the $\mathrm{Nb}_{3} \mathrm{Si}$ transition temperature. Inset: The normalized differential resistance verses bias at $1.6,1.2$ and $0.35 \mathrm{~K}$. The 1.2 and $1.6 \mathrm{~K}$ traces are offset by 0.1 and 0.2 , respectively. The gap widths for $\mathrm{Nb}$ and $\mathrm{Nb}_{3} \mathrm{Si}$ are indicated.

FIG. 3. (a) Four-terminal measurement of the DC current and voltage across the Nb/Si interface at $T=0.35 \mathrm{~K}$ and $T=1.6 \mathrm{~K}$. Each trace shows measurements made at 1000 equally spaced values of the applied bias $V_{a}$ (see inset). The dashed line shows the characteristics for $B=$ $1.0 \mathrm{~T}$ and $T=0.35 \mathrm{~K}$. (b) DC current and voltage characteristics plotted for 26 equally spaced temperatures between $0.35 \mathrm{~K}$ and $1.6 \mathrm{~K}$. The traces are offset for clarity. Inset The normalized switching bias as a function of reduced temperature for the three switches indicated.

FIG. 4. The normalized switching bias as a function of (a) reduced temperature and (b) magnetic field for a typical resistance switch. 
This figure "Fig1.GIF" is available in "GIF" format from: http://arxiv.org/ps/cond-mat/9707071v2 
This figure "Fig2.GIF" is available in "GIF" format from: http://arxiv.org/ps/cond-mat/9707071v2 
This figure "Fig3.GIF" is available in "GIF" format from: http://arxiv.org/ps/cond-mat/9707071v2 
This figure "Fig4.GIF" is available in "GIF" format from: http://arxiv.org/ps/cond-mat/9707071v2 